\title{
IN MEMORY \\ OF KOSTYANTYN PAVLOVYCH SHAMRAI
}

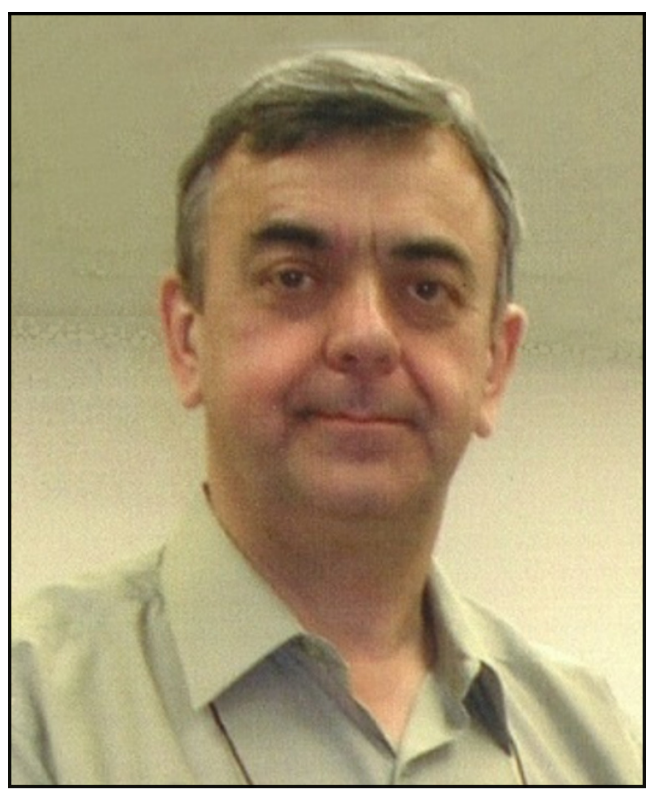

On September 6, 2013, Kostyantyn Pavlovych Shamrai, the known physicist-theorist in plasma physics, Dr. Sci. in physics and mathematics, the Head of Plasma Theory Department at the Kyiv Institute for Nuclear Research (KINR) of the National Academy of Sciences of Ukraine suddenly passed away at the age of 63 .

Kostyantyn Pavlovych began his scientific activity in 1972 at the INR of the Academy of Sciences of the Ukrainian SSR (now, KINR of the National Academy of Sciences of Ukraine), where he worked his way up from postgraduate to Head of the Department.

A keen feeling of all new was always inherent to K.P. Shamrai. The scope of his scientific interests included the most challenging problems of modern plasma physics. First of all, these were the nonlinear wave processes in nonequilibrium and inhomogeneous plasmas and the mechanisms of high-frequency power absorption in helicon plasma. Possessing a brilliant talent of physicist-theorist, K.P. Shamrai penetrated deeply into the essence of physical experiments and clearly saw the application prospects for fundamental phenomena.

In the 1980s and at the beginning of the 1990s, K.P. Shamrai obtained a series of important results in the theory of the interaction of electromagnetic radiation and charged-particle beams with plasma. He studied the processes of wave transformation and amplification in inhomogeneous plasma with an electron beam, discovered the effect of nonlinear saturation of the resonance absorption of surface waves in plasma, proved that this effect can be used to enhance the efficiency of electron beam energy absorption, and analyzed the influence of an external high-frequency field on the evolution of the beam instability in plasma.

K.P. Shamrai showed off his creative talent, while studying a helicon discharge and the properties of helicon plasmas, as well as while developing devices and technologies on their basis for various application. Here he obtained most important results that became widely known in the world and got appreciation and recognition of his colleagues. In particular, he developed the theory of the collective absorption of high-frequency power by linear conversion of helicon waves to a Trivelpiece-Gould wave. He found that the jumps of the plasma density in helicon plasma sources are caused by the non-monotonic dependence of the plasma resistance on the plasma density, and that they are inherent to helicon discharges. As a result, he proposed ways to minimize this phenomenon. He showed that a flux of fast electrons can be formed near the metal surfaces of a discharge system owing to the stochastic acceleration, and studied a lot of other effects.

The theories developed by K.P. Shamrai explain the physical phenomena experimentally observed at

ISSN 2071-0194. Ukr. J. Phys. 2013. Vol. 58, No. 12 
the KINR and foreign laboratories. His theoretical works provide a basis for the creation of new devices based on helicon discharges, which can be applied under terrestrial and space conditions. K.P. Shamrai actively cooperated with scientists from Japan, USA, Russia, Europe, and Australia. He honorably represented the Ukrainian science throughout the world. For the cycle of works "Studies of physical phenomena in dense helicon plasma and their application", the Minister of Education, culture, sports, science, and technologies of Japan awarded K.P. Shamrai together with S. Shinohara (Kyushu University, Fukuoka, Japan) and T. Tanikawa (Tokai University, Kanagawa, Japan) a prize for achievement in science and engineering in 2009.

K.P. Shamrai carried out an active pedagogical, scientific-managerial, popularization, and social work. He lectured at the Taras Shevchenko National University of Kyiv, was the scientific secretary of the Scientific council board in the problem "Plasma physics and plasma electronics" of the National Academy of Sciences of Ukraine, and a member of a number of specialized council boards. He trained a num- ber of young scientists, theorists and experimenters, in plasma physics.

His modesty, goodwill, profound knowledge, wide erudition, responsiveness, and decency resulted in a deep respect and love to Kostyantyn Pavlovych from his relatives, friends, and colleagues. His premature death is a heavy loss to science and the scientific community. The blessed memory of Kostyantyn Pavlovych Shamrai will forever be kept in the heart of everyone who knew him.

M.O. AZARENKOV, I.M. VYSHNEVSKYI, A.G. ZAGORODNY, V.A. ZHOVTYANSKY, V.I. SLISENKO, I.O. ANISIMOV A.G. BORISENKO, V.F. VIRKO, I.E. GARKUSHA, O.A. GONCHAROV, D.L. GREKOV, A.A. GURIN, V.YU. DENISOV, V.I. ZASENKO, V.I. KARAS', Y.I. KOLESNICHENKO, V.M. LASHKIN, I.M. ONISHCHENKO, V.M. PAVLENKO, V.M. SLOBODYAN, P.D. STARCHYK, O.A. FEDOROVYCH, V.V. TSIOLKO 